\title{
Proceedings of the 143rd Semon Club, 15 May 2012, ENT Department, Guy's and St Thomas' NHS Foundation Trust, London, UK
}

\author{
Chairman: Miss Elfy B Chevretton, Guy's and St Thomas' NHS Foundation Trust \\ Secretary: Mr Sherif Haikel, Guy's and St Thomas' NHS Foundation Trust \\ Invited panel for pathology: Dr Ann Sandison, Imperial College Healthcare NHS Trust, Charing Cross Hospital \\ Invited panel for radiology: Dr Steve Connor, and Dr Ata Siddiqui, Guy’s and St Thomas' NHS Foundation Trust
}

The Professor Leslie Michaels prize for the best presentation of the meeting was awarded to D Allin for 'A novel cause of rhinosinusitis: a rare cause of sinonasal tumour'

\author{
Otology section \\ Chairman: Mr D Jiang
}

A case of ageusia, hyperacusis and preserved facial nerve motor function following head injury

\section{T Ashfield, R Obholzer}

From Guy's Hospital, London

A 22-year-old woman presented to the emergency ENT clinic, 10 days following a head injury sustained from a $1 \mathrm{~m}$ fall. She was admitted under the neurosurgical team for 2 days of observation, with evidence of a left lateral temporal bone fracture.

Computed tomography revealed a contusion to the left gyrus rectus, and clear external auditory meati and middleear cavities, with no ossicular disruption.

On assessment in clinic, she complained of ageusia, anosmia and hyperacusis since the event. She was otherwise recovering well and denied any subjective hearing loss or vertigo. On examination, the left ear canal was oedematous with evidence of a haemotympanum behind an intact tympanic membrane. There was no evidence of nasal or otic cerebrospinal fluid leakage. Tuning fork tests were normal and facial nerve motor function was intact. Taste testing revealed absent taste sensation to co-phenylcaine on the left side of the tongue but preserved on the right. Cranial nerve examination was otherwise normal. The taste of co-phenylcaine in the throat was reported after administration to the nose. Flexible nasendoscopy revealed no abnormality.

Audiometry demonstrated normal hearing and type A tympanograms bilaterally. A stapedial reflex was detected on the right, but despite recurrent testing there was an equivocal response on the left.

This case was consistent with traumatic injury to the left chorda tympani and possibly the nerve to the stapedius. Motor facial nerve function was preserved. Dr Connor noted a longitudinal fracture of the left temporal bone, not tracking through the course of the chorda tympani but close to it. This case was being managed conservatively at the time of writing, pending discussion at the skull base multidisciplinary team meeting.
An unusual cause of right pulsatile tinnitus and left ophthalmoplegia

H Dixon, B Fu, S Watts

From the Royal Sussex County Hospital, Brighton

Background

This case highlights an unusual presentation of a combination of pathologies, with multidisciplinary team management.

\section{Case report}

A 59-year-old man presented with frontal headaches, right pulsatile tinnitus and vertigo. Examination demonstrated a left trochlear nerve palsy, left ptosis and pulsatile right middle-ear 'glue'. Past history included previous right mastoiditis. Initial treatment included right myringotomy and grommet insertion plus post-nasal space biopsy, together with antibiotics. The patient was reviewed by ophthalmology. Subsequent magnetic resonance imaging (MRI) of the head showed an orbital soft-tissue mass which had not been present in previous scans. Endoscopic biopsy was performed.

\section{Histopathology}

The post-nasal space biopsy was unremarkable. The orbital biopsy showed a fibroblastic-myofibroblastic tissue consistent with an orbital pseudotumour.

\section{Radiology}

Computed tomography of the head showed increased opacification of the right mastoid air cells, in keeping with previous mastoiditis. The initial MRI of the head excluded a skull base lesion. Repeated MRI showed abnormal soft tissue extending into the left globe and causing proptosis. Dr Connor also suggested the possibility of an abnormality in the left frontal lobe.

\section{Management}

Initially, a post-nasal space lesion extending up to cause skull base pathology was considered. It was only on repeated MRI and clinical review that the progression of disease was demonstrated. After biopsy of the mass suggested an orbital pseudotumour, the patient was reassured and commenced on oral steroids. 


\section{Discussion}

Dr Sandison agreed with the histopathological opinion sought from the USA, which suggested further studies were required to rule out other inflammatory pathologies (e.g. Wegener's vasculitis). Dr Connor suggested that a high resolution, fine cut MRI scan would help to determine the true margins of the disease; this has since been requested as per his advice.

\section{An unusual swelling of the ear canal}

\section{A Alatsatianos, C Theokli, P Gluckman \\ From Medway NHS Foundation Trust, Gillingham}

Follicular lymphoma is the most common type of nonHodgkin's lymphoma and can arise from lymph nodes or from extra-nodal sites. It is very uncommon for lymphoma to arise from the ear canal.

An 88-year-old woman was referred to the ENT rapid access clinic with a history of unilateral otalgia for four months and a transilluminating, cystic swelling arising from the postero-superior wall of the right ear canal. She also suffered from cataracts and ocular hypertension, and was diagnosed and successfully treated for ovarian carcinoma during investigation for her ear swelling.

Computed tomography of the mastoids displayed a normal middle ear and mastoid air cells. Magnetic resonance imaging of the head revealed that the mass was confined to the ear canal and had no communication with the brain, excluding a meningocoele. Signal from this mass was not suppressed on fluid attenuated inversion recovery ('FLAIR') images, suggesting a proteinaceous cyst.

Examination of fine needle aspirate identified lymphocytes but no noticeable fluid.

The patient underwent excision of the right ear canal mass. Histological examination of the specimen revealed a nonHodgkin's B-cell lymphoma with the immunophenotype and morphology of a follicular lymphoma (World Health Organization grade 1). Haematology input ruled out a primary source elsewhere, and the final diagnosis was primary cutaneous follicular lymphoma grade 1A of the right ear canal. The patient subsequently underwent radiotherapy and was disease-free at the time of writing.

This case illustrates a very rare presentation of primary cutaneous follicular lymphoma. After reviewing the available literature on follicular lymphoma, this patient may represent the first described case of its type.

Dr Sandison reminded the meeting that the role of surgery in the management of these lesions is limited to biopsy for histology.

Unilateral sensorineural hearing loss, tinnitus and episodes of vertigo

J Virk, P Randhawa, S Saeed

From the Royal National Throat, Nose and Ear Hospital, London

\section{Background}

Endolymphatic sac tumours are extremely rare, and can be sporadic or found in association with von Hippel-Lindau disease. They are thought to emanate from the intratemporal portion of the endolymphatic sac. They are slow growing, locally destructive and difficult to diagnose due to their initial asymptomatic phase and wide array of differential diagnoses.
Case report

A 79-year-old man presented with unilateral right-sided hearing loss, tinnitus and episodes of vertigo over a sixmonth period. Examination revealed an intact right tympanic membrane with a non-pulsatile, red-blue mass behind it. The left ear was normal. The facial nerve was intact. Audiometry confirmed a profound sensorineural hearing loss on the right, with normal thresholds on the left.

\section{Radiological findings}

Computed tomography and magnetic resonance imaging of the temporal bones elucidated a destructive lesion involving the petrous apex and extending into the posterior semicircular canal. No dural breach was evident.

\section{Histological findings}

Histology confirmed sections of fibrous tissue and bone showing infiltration by bland papillary neoplasm.

\section{Management}

A transmastoid translabyrinthine excision was performed with the use of a post-aural extended cortical mastoidectomy incision. The tumour reached the common crus from the posterior semicircular canal but spared the jugular bulb, and was completely excised macroscopically. The patient recovered well with normal facial function.

\section{Discussion at meeting}

Drs Connor and Sandison reviewed the differential diagnosis and opined that, although difficult to diagnose, the classical radiological and histological findings were displayed in this case. Dr Sandison commented that, due to the paucity of cases, management is not standardised, and histology should be reviewed in a specialist centre.

Congenital fistula between the second and fifth hillocks of His: a unique otological anomaly?

J Siddiqui, V Prasad, K Seymour

From Barts Health NHS Trust, London

\section{Background}

Auricular development is complex and begins as six mesenchymal buds, the hillocks of His, which form the anatomical components of the developed external ear. Congenital anomalies resulting from pathology of this developmental pathway have been described theoretically, and some documented clinically. We present the case of a congenital fistula between the second and fifth hillocks of His, the embryological precursors of the helical crus and antihelix, respectively. To our knowledge, this is the first such documented case.

\section{Case report}

A two-year-old boy presented with recurrent external ear infections, treated as furunculosis. At our clinic, a cleft on the right helical crus was seen to communicate with the posterior external auditory canal wall.

\section{Radiological findings}

A magnetic resonance imaging (MRI) scan of the region confirmed this anomaly to be unilateral and isolated, with no associated first branchial cleft abnormality.

\section{Management and histological findings}

Surgical excision of the tract confirmed it clinically as a fistula, between the derivatives of the second and fifth 
hillocks of His, an anomaly previously only described theoretically. Histology was in concordance with these findings. The patient made a complete recovery, with no further infection.

\section{Discussion}

Most agreed at the meeting that such anomalies may often go undiagnosed, treated as recurrent infection. Clinical suspicion of such an anomaly mandates MRI scanning to exclude associated branchial cleft anomalies and bilateral pathology.

\section{Conclusion}

This is the first documented case of such an anomaly. A high index of suspicion and low threshold for MRI scanning are necessary to avoid a missed diagnosis.

\section{Rhinology section}

Chairman: Miss C Hopkins

A rare case of unilateral nasal obstruction and swelling

B Cadd, S Latis, G Alusi

From Barts and The London NHS Trust, London

A 31-year-old man was referred to the ENT clinic with a 3-month history of nasal obstruction and swelling. During the evolution of this mass, he had also experienced sporadic episodes of mild epistaxis. Examination revealed a large, right-sided nasal polyp which was also expanding the lateral wall of the nose. Magnetic resonance imaging revealed a right nasal lesion of $3.3 \mathrm{~cm}$ diameter extending through the cartilage of the nasal septum and into the submucoperiosteal space of the contralateral side of the nasal septum.

Differential diagnoses included haemangioma and pyogenic granuloma. The lesion was biopsied in the operating theatre; histopathological examination revealed mixed Antoni A and B type histology, and strong S100 staining, giving a diagnosis of nasal septal schwannoma. The patient underwent a mid-facial degloving procedure with excision of the tumour, which was confirmed to be a septal schwannoma with diffuse, moderate intensity positivity for S100 staining amongst the neoplastic cells.

Nasal septal schwannoma is an exceptionally rare presentation of a well understood pathological process. There are only 20 other cases reported in the literature. A thorough analysis of the radiological appearance of nasal septal schwannoma has yet to be published.

An unusual cause of unilateral middle-ear effusion and facial paraesthesia

B Wong, B Fu, M Oyarzabal

From Medway Maritime Hospital, Gillingham

\section{Background}

Extramedullary plasmacytoma is a rare neoplasm of plasma cells, which may occur as part of a generalised disease or as a local entity. These lesions make up less than 1 per cent of all head and neck malignancies and have a predilection for the nasal airways.

\section{Case report}

A 63-year-old woman presented with left-sided deafness due to a middle-ear effusion, together with preauricular facial paraesthesia. Grommet insertion and post-nasal space biopsy were performed. Due to persistent post-operative facial symptoms and subsequent development of generalised malaise, computed tomography (CT), magnetic resonance imaging (MRI) and an endonasal biopsy were also performed.

\section{Radiology}

The CT showed an erosive lesion involving the left maxillary antrum, sphenoid bone, and infratemporal and pterygoid fossae. Additional lytic lesions were seen in the sternum and left ninth rib. The MRI confirmed a $5-\mathrm{cm}$ soft tissue mass centred in the left masticator space and eroding into the posterior wall of the maxillary antrum. The mass also extended into the left pterygopalatine fossa, inferior orbital fissure, foramen ovale, left cavernous sinus and clivus.

\section{Histopathology}

Histopathological analysis of the biopsy showed a tumour formed of a diffuse proliferation of plasma cells in various stages of maturation, including binucleated and occasional multinucleated forms. Immunohistochemical analysis showed strong staining for the cluster of differentiation 138 and 20 protein markers, and also showed clonal restriction for lambda light chains. These findings were in keeping with a plasmacytoma or multiple myeloma.

\section{Management}

Histology and radiological results were discussed at the head and neck and haematology multidisciplinary team meetings. Treatment with chemotherapy and radiotherapy to the left maxilla was planned.

\section{Discussion}

Both Dr Sanderson and Dr Connor concurred with the local histology and radiology report.

\section{Conclusion}

Extramedullary plasmacytoma can present with unilateral middle-ear effusion and facial paraesthesia.

\section{An unusual cause of periorbital swelling}

A Ramessur, N Hamilton, A Hannan

From the Royal Free Hospital, London

\section{Introduction}

We present an 81-year-old woman with periorbital swelling which proved to be a diagnostic challenge.

\section{Case report}

The patient presented with pyrexia, confusion and rightsided periorbital swelling refractory to antibiotic treatment. Computed tomography (CT) demonstrated opacification of the ethmoid sinuses and a subperiosteal lesion in the right orbit. The patient underwent emergency naso-endoscopic decompression of the right medial orbital wall and external incision and drainage via a Lynch-Howarth approach. Pus and inflammatory tissue were sent for microbiology and histology. Despite intravenous antibiotics, the patient continued to deteriorate, and a second CT showed a persistent subperiosteal collection. The patient was returned to the operating theatre but no recurrent collection was found.

\section{Radiological findings}

The first CT of the sinuses (taken on admission) showed acute rhinosinusitis complicated by a right medial 
subperiosteal orbital mass, probably an abscess, tracking posteriorly and threatening the optic nerve.

The second sinus CT showed a persistent subperiosteal mass with ongoing displacement of the right globe and medial rectus.

\section{Histological findings}

Upon histopathological analysis, a specimen from the right ethmoid sinus showed necrotic tissue with marked apoptosis and cellular debris within the lamina propria, interspersed with numerous atypical cells with irregular, hyperchromatic nuclei and increased mitoses. The diagnosis was confirmed as extranodal natural killer cell/T-cell lymphoma.

\section{Management}

In view of the aggressive nature and poor prognosis associated with natural killer cell/T-cell tumours, and the patient's evidence of distant metastases, the multidisciplinary team made the decision to proceed with palliative treatment.

\section{Conclusion and lessons learned}

Dr Connor held the opinion that radiological contrast studies may have been useful. This case highlights the importance of considering other differentials when faced with a case of refractory periorbital swelling, and also emphasises the importance of sending samples for histology during surgery.

\section{A novel cause of rhinosinusitis: a rare cause of sinonasal tumour}

D Allin, J Stephens, W Grant

From Charing Cross Hospital, London

\section{Introduction}

Hyper immunoglobulin G4 related disease is a recently described auto-immune disorder with multisystem manifestations. Involvement of the ethmoid or sphenoid sinuses has not previously been described in the literature; thus, the patient described below represents a novel clinical entity.

\section{Case report}

A 57-year-old man presented with a one-year history of bilateral submandibular swelling, cervical lymphadenopathy and symptoms of chronic rhinosinusitis.

\section{Radiological findings}

Computed tomography (CT) of the sinuses revealed soft tissue in the nasal vault bilaterally, consistent with atypical sinonasal polyposis. There was complete opacification of both frontal and sphenoid sinuses and ethmoid air cells, together with moderate mucosal thickening in both maxillary sinuses.

\section{Histological findings}

Analysis of intra-operative samples from the left ethmoid sinus revealed oedematous, fibrotic respiratory mucosa with a lymphoplasmacytic and eosinophilic inflammatory infiltrate. Subsequent staining showed that the immunoglobulin (Ig) G4/IgG ratio was approximately 1, confirming the diagnosis of hyper-IgG4 disease.

\section{Management}

The patient was commenced on oral steroids and showed a marked improvement in symptoms.

\section{Discussion}

This is the first reported case of hyper IgG4 related disease involving the ethmoid and sphenoid sinuses. Our presentation of this case aims to raise awareness of the possibility of hyper-IgG4 disease occurring in the nasal cavity, and to propose that this condition be included in the differential diagnosis of non-malignant nasal tumours. Miss Hopkins was of the opinion that this case illustrated how a novel diagnosis could present with relatively common CT scan findings, and reminded the meeting of the importance of taking multiple endoscopic biopsies to aid accurate diagnosis.

\section{A rare case of sinonasal mass presenting with cranial nerve neuropathy}

\author{
S Davey, S Baer \\ From Conquest Hospital, St Leonards-on-Sea
}

\section{Background}

We present a case of breast carcinoma metastasis to the nasopharynx and paranasal sinuses. To date, there have only been two documented cases in the English language literature.

\section{Case report}

A 75-year-old woman presented with signs and symptoms of primary sinonasal malignancy. She had been referred to ENT by the ophthalmologists, who had organised a magnetic resonance imaging (MRI) scan to investigate a left abducens nerve palsy and diplopia. Flexible nasendoscopy revealed a lobulated swelling in the left post-nasal space.

\section{Radiology}

The MRI scan revealed no intra-cranial cause for the patient's initial symptoms; however, a $2.7 \times 3.2 \times 2.6 \mathrm{~cm}$ soft tissue mass was demonstrated around the ethmoid and sphenoid sinuses.

\section{Histopathology}

The original biopsy from the endoscopy clinic showed a polypoid mass of complexly folded, broad trabeculae of squamous epithelium, with numerous acute inflammatory cells present. The initial tissue diagnosis was inverted sinonasal papilloma. However, this diagnosis did not correlate with the clinical presentation, so a second biopsy was performed, which confirmed metastatic breast cancer and was oestrogen receptor positive.

\section{Management}

Unfortunately, the patient deteriorated rapidly and was managed palliatively. Truly isolated lesions, i.e. those without extensive regional invasion or disseminated disease, may be managed by primary surgical resection.

\section{Discussion and lessons learned}

Although initial histological analysis was inconclusive, Dr Sandison stated that malignancy could not be ruled out and that signs of malignancy were present. Notably, the second biopsy confirmed metastatic breast cancer and was oestrogen receptor positive. Following discussion, the consensus of the meeting was that this entity was indeed a rare occurrence. A high index of suspicion should always be employed in cases with a past history of malignancy. 


\section{Paediatric section}

Chairman: Mr Ian Hore

A child with bilateral conductive hearing loss and sudden onset of unilateral facial weakness

\section{A Khan, P Stimpson, N Patel}

From Whipps Cross University Hospital NHS Trust, London

\section{Introduction}

We present a unique case of middle-ear involvement in a child with Gaucher's disease, a rare, autosomal recessive, lysosomal storage disease. This patient represents the first reported case of Gaucher's disease affecting the middle ear and mastoid in the world literature.

\section{Case report}

A five-year-old boy with known Gaucher's disease presented with bilateral hearing impairment and a conductive hearing loss on pure tone audiometry, with flat tympanometry traces. During a period of 'watchful waiting', the child developed a grade 3 , right-sided facial weakness following an episode of acute otitis media. The facial weakness resolved spontaneously; placement of grommets was attempted but could not be completed due to the presence of vascular, friable material filling both middle ears. Imaging was subsequently performed. The child underwent exploratory tympanotomy and cortical mastoidectomy. Large amounts of inflammatory material were cleared from the mastoid and middle ear.

\section{Radiological findings}

Computed tomography of the temporal bones demonstrated extensive opacification of the middle ear and mastoid bilaterally, with no evidence of bony erosion.

\section{Histological findings}

Specimens from the middle ear demonstrated histiocytes with abundant, fine fibrillary, pale grey cytoplasm with small, peripherally located, indistinct nuclei with coarse chromatin. The findings were suggestive of Gaucher's disease within the middle ear.

\section{Management}

At the time of writing, the child continued under multidisciplinary care for his Gaucher's disease, with renewed focus on auditory rehabilitation.

An infant presenting with acute loss of vision and a rare sinonasal mass

\section{Pepper, D Tweedie, L Cochrane}

From Great Ormond Street Hospital, London

\section{Introduction}

Sinonasal malignancies in children are very unusual, particularly in those under one year of age. The differential diagnosis includes tumours with small, round, blue cells on microscopy and staining, as seen in the presented case. The presenting feature of bilateral visual loss has not previously been described in an infant with sinonasal Ewing's sarcoma.

\section{Case report}

An eight-month-old boy attended the accident and emergency department with a 5-day history of reduced vision. The child was otherwise well, with no nasal or intracranial symptoms. The ophthalmology team arranged an urgent magnetic resonance imaging (MRI) scan, which revealed a sinonasal mass, and referred the patient to ENT. A computed tomography (CT) scan was performed, followed by endoscopic nasal biopsy. Dexamethasone was commenced early to minimise peri-tumour oedema of the optic nerves.

\section{Radiological findings}

Magnetic resonance imaging and CT showed a large, infiltrative, destructive tumour centred on the sphenoid sinus and invading the skull base, orbital cavities and left optic nerve. A chest CT revealed no evidence of metastasis.

\section{Histological findings}

Microscopy of biopsy specimens showed a tumour composed of small, round, blue cells, with positivity for cluster of differentiation 99 and 56 proteins, and local vascular and bone infiltration. In addition, fluorescence in-situ hybridisation was positive for Ewing sarcoma breakpoint 1 gene protein, confirming the diagnosis of Ewing's sarcoma.

\section{Management}

The child was discussed at the multidisciplinary team meeting and treated with chemotherapy. Magnetic resonance imaging showed a tumour response, with reduction in size particularly in the left cavernous sinus. Unfortunately, the child's vision did not recover.

\section{Unilateral proptosis and diplopia in a six-year-old girl \\ C Sellers, C Hopkins \\ From Guy's and St Thomas' NHS Foundation Trust, London}

\section{Background}

Fronto-ethmoidal mucoceles are benign, cystic lesions caused by obstruction of the mucus flow of the paranasal sinuses. They are capable of invading surrounding structures by dynamic bony resorption and remodelling. Symptoms depend on the orientation of expansion and pressureerosion of the cyst, and range from diplopia and proptosis to facial pain, nasal obstruction and headache. The object of therapy is complete cyst excision and restoration of mucus drainage, typically by external fronto-ethmoidectomy. Advances in functional endoscopic sinus surgery have made this approach more prevalent. Here, we describe endoscopic drainage of a large fronto-ethmoidal mucocele in a six-year-old, with complete symptomatic and cosmetic resolution.

\section{Case report}

A six-year-old girl presented with three weeks of progressive right proptosis and diplopia. Examination revealed significant infero-lateral displacement of the right orbit and a bulging fullness into the right middle meatus. A large fronto-ethmoidal mucocele was diagnosed on imaging. The child rapidly underwent successful endoscopic drainage.

\section{Radiological findings}

Computed tomography and magnetic resonance imaging of the brain and orbit showed marked expansion of the right fronto-ethmoidal sinus by the mucocele, with expansion of the medial orbital wall.

\section{Management}

Endoscopic drainage with antrostomy was performed to prevent ostial obstruction by soft tissue prolapse. Post-operative review revealed a large opening into the 
ethmoids with minor polypoid change of the lining, and free drainage.

\section{Discussion}

Mr Hore enquired as to the technical feasibility of endoscopic surgery in paediatric patients, and was reassured that the relative dimensions of nasal anatomy permitted excellent access to the paranasal sinuses in experienced hands. It was concluded that endoscopic surgery provides a safe and practical alternative to open excision of paranasal mucoceles in children.

\section{Congenital nasal airway obstruction: a familial case series}

\section{S Abdalla, O Khan, C Hopkins}

From Guy's and St Thomas' NHS Foundation Trust, London

\section{Introduction}

Choanal atresia is a rare but significant congenital defect because of its ability to interfere with normal respiratory function. Here, we describe two familial cases of choanal atresia.

\section{Case report and radiological findings}

A male infant born at term by elective caesarean section developed acute respiratory distress during his first feed. Nasogastric tubes could not be passed through either nostril. Computed tomography of the sinuses, performed three days after birth, confirmed bilateral bony and membranous choanal atresia.

Some years later, the mother of the infant presented with long-standing, right-sided nasal obstruction with sinus discomfort and purulent discharge. She was known to suffer from right-sided eustachian tube dysfunction. Computed tomography of her sinuses revealed polypoidal soft tissue occluding the right posterior nasal cavity. A diagnosis of unilateral (right) membranous choanal atresia was made.

\section{Management}

Five days after birth, the infant successfully underwent endoscopic perforation of the posterior choanae, with stent insertion. Subsequently, he required repeated, elective revision and dilatation procedures, predominantly of the right posterior choana, which had a tendency to re-stenose. The mother's unilateral membranous choanal atresia was surgically corrected, and she too required further surgery due to re-stenosis.

\section{Discussion}

This report presents examples of bilateral and unilateral varieties of choanal atresia in successive generations. Another child in the presented family had had unilateral choanal atresia and died in infancy. Choanal atresia in siblings has been described in the literature; however, to the best of our knowledge, this is the first report of unilateral and bilateral choanal atresia in siblings and successive generations. Dr Connor reminded the audience of the 'CHARGE' syndrome (i.e. coloboma of the eye, heart defects, atresia of the nasal choanae, retardation of growth and/or development, genital and/or urinary abnormalities, and ear abnormalities and deafness), in which choanal atresia and ear abnormalities coexist. He also advised that, whenever choanal atresia is diagnosed on a scan, the presence of the semicircular canals should also be confirmed on the same scan.
Unilateral, painless, slow-growing facial mass in a child

L Jablenska, A Trinidade, P Kothari

From Luton and Dunstable University Hospital, Luton

\section{Case report}

A seven-year-old, fit and well girl presented with a twomonth history of a painless, left-sided mass over the parotid area. The mass had been noticed incidentally by her mother. There were no other associated symptoms. Examination revealed a $1.5 \times 1.5 \mathrm{~cm}$, firm swelling within the left parotid. The rest of the ENT examination was unremarkable.

\section{Radiological findings}

An ultrasound scan confirmed the presence of a well-defined, $1.5 \times 2 \mathrm{~cm}$, predominantly solid lesion with minor internal cystic spaces arising from the superficial part of the parotid gland. A magnetic resonance imaging (MRI) scan was used for tumour mapping, and showed no invasion of the deep lobe or skeletal erosion.

\section{Management}

Controversially, fine needle aspiration cytology was not performed for this patient. Superficial parotidectomy was performed via a facelift incision. Post-operatively, there was an excellent cosmetic outcome. At the time of writing, follow up had been planned for three months postoperatively.

\section{Histological findings}

Histopathology following superficial parotidectomy confirmed a pleomorphic adenoma with no evidence of malignancy. However, the lesion extended to one resection margin, where the lesion was completely encapsulated.

\section{Conclusion}

To the best of our knowledge, this is the youngest reported case of pleomorphic adenoma in the literature. Dr Connor commented that, on MRI, the lesion appeared partially encapsulated with cellular and myxoid areas, in keeping with a pleomorphic adenoma. Dr Sandison agreed that cytology was not required pre-operatively as, whatever the cytology findings, the surgeon is best advised to excise the lesion in case it is a mucoepidermoid tumour.

\section{Head and neck section \\ Chairman: Mr J P Jeanon}

\section{Post-irradiation angiosarcoma of the tongue base}

S Barbur, P D R Spraggs, S J Whitaker

From the Royal Surrey County Hospital, Guildford

\section{Case summary}

A 65-year-old man underwent total mucosal irradiation in 2001 as part of treatment for a tumour 0 node 2 a metastasis 0 stage squamous cell carcinoma of the right side of the neck from an unknown primary. He was a known smoker and had a history of rheumatoid arthritis. In 2008, he re-presented with a short history of a sore throat, hoarseness, dysphagia and weight loss. Examination was unremarkable but examination under anaesthesia showed a bulky tongue base tumour, from which biopsies were taken. A magnetic resonance imaging (MRI) scan was not helpful. Histology with 
immunocytochemistry confirmed the findings of an angiosarcoma.

\section{Radiology}

The MRI showed diffuse, asymmetrical thickening of the oropharyngeal mucosa but no obvious tumour.

\section{Histopathology}

Histopathological examination of the original lymph node specimens illustrated moderately differentiated squamous cell carcinoma. Biopsies of the tongue base, in 2008, were positive for cluster of differentiation 31 protein, a vascular marker, and negative for epithelial markers.

\section{Management}

At the multidisciplinary team meeting, it was decided to treat the patient palliatively. The patients' condition deteriorated rapidly, and he died soon after his diagnosis was confirmed.

\section{Discussion}

Mr Jeanon noted that the expression of cluster of differentiation 31 protein in the second tumour confirmed that it was a second primary vascular tumour, as opposed to a recurrence of the original tumour. He commented that when a case is labelled as having an 'unknown primary', the primary is often located in the tongue base or the base of the tonsil. He also commented on the risks of total mucosal irradiation and the long term risks of resulting complications.

Weight loss, dysphagia and hypernasality in a retired painter and decorator

\section{K Andi, R Simo}

From Guy’s Hospital, London

\section{Introduction}

Submucosal tumours associated with a reduction of the nasooropharyngeal airway are uncommon and often present late once symptoms have ensued. The differential diagnosis includes squamous cell carcinoma, lymphoma and sarcoma as well as rare tumours originating from the cervical spine.

\section{Case report}

A 69-year-old, retired painter and decorator presented to the head and neck department with a 3-month history of dysphagia to solids. During this time, his daughter had noticed a change in his voice.

Examination identified a $2.5 \mathrm{~cm}$, left-sided, level II lymph node. Intraorally, the soft palate and uvula were displaced anteriorly, restricting the view of the pillar of fauces particularly on the left side. A flexible nasoendoscope could not be passed beyond the postnasal space, which was significantly narrowed by the protuberance of the posterior pharyngeal wall mucosa.

\section{Radiological findings}

A mass was evident originating from the bodies of the second and third cervical vertebrae, with destruction of the vertebral bodies and soft tissue extension into the prevertebral space. Both vertebral arteries were intimately associated with the tumour.

\section{Histological findings}

Histological examination identified features consistent with a classical chordoma.

\section{Management}

The patient underwent transoral excision of the tumour, facilitated by cervical spine fixation. Access was gained via a lip-split mandibulotomy with midline tongue, floor of mouth and soft palate division, as well as vertical pharyngotomy and tracheostomy.

An enlarging central neck mass following renal cell carcinoma metastasis to the parathyroid gland

\section{Rishi, O Enyi, R Simo}

From Guy's Hospital, London

\section{Background}

Renal cell carcinoma has a tendency to metastasise in an unpredictable fashion. Renal cell carcinoma metastasis to the parathyroid glands is exceptionally rare, and to our knowledge has never been reported in the literature. We present a case of renal cell carcinoma metastasis to the parathyroids, together with a review of the literature.

\section{Case report}

A 69-year-old, Caucasian man underwent a right radical nephrectomy for a right kidney renal cell carcinoma. Subsequent follow-up appointments and cross-sectional imaging showed no abnormalities. However, an 11-mm, solitary lesion was later observed in the right paraesophageal area on computed tomography scanning. This enhancing node adjacent to the oesophagus gradually increased in size to $15 \mathrm{~mm}$, prompting referral to ENT. Excision of the neck mass via a cervical approach was performed and the sample sent for histopathological analysis. The excision biopsy was reported as showing metastatic renal cell carcinoma to the right parathyroid gland.

\section{Conclusion}

It is rare for a patient to undergo head and neck surgery secondary to renal cell carcinoma metastasis. It is exceptionally rare for a patient to require head and neck surgery due to a renal cell carcinoma metastasis to the parathyroid gland.

Odynophagia with cervical lymphadenopathy in a patient previously treated with radiotherapy for lymphoma

C Seung-Jin, K Sahr, S Sood

From Bradford Royal Infirmary

We present a rare case of a patient with tumour to tumour metastasis of head and neck cancer.

An 84-year-old, retired banker presented with a 6-month history of odynophagia, pain in the base of the tongue, and bilateral, level II lymphadenopathy. He was an ex-smoker and drank alcohol moderately. He had a previous history of mantle cell lymphoma in 1998, for which he had received chemo-radiotherapy.

Clinical examination revealed bilateral, level II lymphadenopathy and an ulcerated right tongue base.

The patient underwent panendoscopy, which revealed a large, exophytic, right-sided tongue base tumour extending into the vallecula, and also a right pharyngeal tumour. Biopsies were taken and cross-sectional imaging performed.

A computed tomography scan of the neck, chest, abdomen and pelvis showed bilateral nodal enlargement in the neck and multiple lymphadenopathies throughout the chest, abdomen and pelvis. 
Biopsies from the right tongue base and right pharynx showed invasive squamous cell carcinoma. Histopathological examination of a lymph node biopsy showed chronic lymphocytic leukaemia infiltrated by metastatic squamous cell carcinoma (SCC).

The patient was discussed at the multidisciplinary team meeting and was treated with palliative radiotherapy.

The panel agreed that chronic lymphocytic leukaemia and head and neck SCC can occur together, but never had they seen these two conditions occurring in the same lymph node.

Tumour to tumour metastasis in head and neck cancer is rare, and poses a diagnostic dilemma of which ENT surgeons should be aware. Dr Sandison suggested that this occurrence may be more common than is suspected and that, microscopically, the chronic lymphocytic leukaemia component may often be missed once the SCC is diagnosed.

Dyspnoea, stridor and wheeze in a young woman

C Stamatoglou, J Virk, G Sandhu

From Charing Cross Hospital, Imperial College Healthcare NHS Trust, London

\section{Introduction}

Immunoglobulin 4 related autoimmune fibrosclerosing pseudotumour is a recently recognised clinical entity with a variety of presentations. It is characterised histopathologically by extensive immunoglobulin (Ig) G4 positive plasma cell infiltration alongside $\mathrm{T}$ lymphocytes. This report describes a novel local manifestation of this systemic disease, in the trachea.
Case report

A 22-year-old woman presented initially in her native Barbados with acute dyspnoea and wheeze, managed as an exacerbation of asthma. She became stridulous and was diagnosed with idiopathic subglottic stenosis, managed with tracheostomy. On review three years later in the UK, she had a small airway and poor exercise tolerance. Having confirmed the presence of benign pathology in the biopsies taken in Barbados, we performed microlaryngoscopy together with laser and balloon dilatation. A firm, amorphous mass was noted intra-operatively and a biopsy taken.

\section{Radiological findings}

Computed tomography imaging revealed abnormal soft tissue extending from the supraglottis to the subglottis, involving the thyroid cartilage and continuing outside the larynx.

\section{Histological findings}

The biopsy exhibited fibrosis together with a dense, acute on chronic, inflammatory infiltrate rich in plasma cells. This was associated with a proliferation of histiocytes positive for cluster of differentiation 68 protein on immunostaining, together with aggregates of mixed cluster of differentiation 20 protein positive B-lymphocytes and cluster of differentiation 3 protein positive T-lymphocytes. The ratio of IgG:IgG4 plasma cells was less than 50 per cent; however, the possibility of an IgG4 sclerosing pseudotumour was raised.

\section{Management}

A laryngotracheal reconstruction was subsequently performed with complete excision of the lesion. The patient recovered well. 